OPEN ACCESS

Edited by:

Viviana Moschese,

University of Rome Tor Vergata, Italy

Reviewed by:

Baldassarre Martire,

Monsignor Raffaele Dimiccoli Hospital,

Italy

Srinivasa Reddy Bonam, Institut National de la Santé et de la

Recherche Médicale (INSERM),

France

*Correspondence: Olufunto A. Olusanya oolusan1@uthsc.edu Arash Shaban-Nejad ashabann@uthsc.edu

Specialty section:

This article was submitted to Vaccines and Molecular Therapeutics, a section of the journal

Frontiers in Immunology

Received: 02 February 2021 Accepted: 04 March 2021

Published: 18 March 2021

Citation:

Olusanya OA, Bednarczyk RA Davis RL and Shaban-Nejad A (2021)

Addressing Parental Vaccine Hesitancy and Other Barriers to

Childhood/Adolescent Vaccination

Uptake During the Coronavirus

(COVID-19) Pandemic.

Front. Immunol. 12:663074. doi: 10.3389/fimmu.2021.663074

\section{Addressing Parental Vaccine Hesitancy and Other Barriers to Childhood/Adolescent Vaccination Uptake During the Coronavirus (COVID-19) Pandemic}

\author{
Olufunto A. Olusanya ${ }^{1 *}$, Robert A. Bednarczyk ${ }^{2}$, Robert L. Davis ${ }^{1}$ \\ and Arash Shaban-Nejad ${ }^{1 *}$
}

1 Oak Ridge National Laboratory, Center for Biomedical Informatics, Department of Pediatrics, College of Medicine, University of Tennessee Health Science Center, Memphis, TN, United States, ${ }^{2}$ Hubert Department of Global Health, Rollins School of Public Health, Emory University, Atlanta, GA, United States

Routine childhood immunizations are proven to be one of the most effective public health interventions at controlling numerous deadly diseases. Therefore, the CDC recommends routine immunizations for children and adolescent populations against vaccinepreventable diseases e.g., tetanus, pertussis, diphtheria, etc. This current review sought to examine barriers to pediatric vaccine uptake behaviors during the COVID-19 pandemic. We also explored the implications for parental vaccine hesitancy/delay during an ongoing health crisis and proposed recommendations for increasing vaccine confidence and compliance. Our review determined that the receipt for vaccinations steadily improved in the last decade for both the United States and Tennessee. However, this incremental progress has been forestalled by the COVID-19 pandemic and other barriers i.e. parental vaccine hesitancy, social determinants of health $(\mathrm{SDOH})$ inequalities, etc. which further exacerbate vaccination disparities. Moreover, non-compliance to routine vaccinations could cause an outbreak of diseases, thereby, worsening the ongoing health crisis and already strained health care system. Healthcare providers are uniquely positioned to offer effective recommendations with presumptive languaging to increase vaccination rates, as well as, address parental vaccine hesitancy. Best practices that incorporate healthcare providers' quality improvement coaching, vaccination reminder recall systems, adherence to standardized safety protocols (physical distancing, hand hygiene practices, etc.), as well as, offer telehealth and outdoor/drivethrough/curbside vaccination services, etc. are warranted. Additionally, a concerted effort should be made to utilize public health surveillance systems to collect, analyze, and interpret data, thereby, ensuring the dissemination of timely, accurate health information for effective health policy decision-making e.g., vaccine distribution, etc.

Keywords: vaccine hesitancy, vaccine confidence, vaccine compliance, vaccine disparity, COVID-19, SARS-COV-2, Social Determinants of Health (SDoH) 


\section{INTRODUCTION}

In the United States (U.S.) and globally, routine prophylactic childhood immunizations are established as public health interventions that are most effective and cost-beneficial at significantly preventing numerous infectious diseases and premature mortalities (1). In the pre-vaccine era and before the 1963 measles vaccination programs, there were roughly 6,000 deaths attributed to the measles virus each year (2). Moreover, between 1964 and 1965, an epidemic of the rubella virus resulted in an estimated 2,000 neonatal deaths and 11,000 fetal miscarriages (3). Accordingly, it has been projected that over 100 million cases of vaccine-preventable illnesses i.e. measles, mumps, rubella, pertussis, etc., have been prevented in the U.S. (4). Between 1994 and 2013, an enormous financial burden to the tune of approximately $\$ 402$ billion and $\$ 1.5$ trillion was prevented in direct and societal costs (5). Concurrently, within Tennessee, a racially and economically diverse state ranking $16^{\text {th }}$ most populous in the U.S, vaccine-preventable diseases have significantly decreased. This is due to widespread institutional policies to increase vaccine uptake (to meet Tennessee Immunization Program (TIP)'s 90\% goal), as well as, wider acceptance of healthcare providers' recommendations (6). Overall, vaccinations continue to serve an essential role in protecting vulnerable individuals from potentially deadly vaccine-preventable illnesses.

Moreover, the scientific community supports the consensus that the highly contagious coronavirus disease 2019 (COVID-19), which was declared a pandemic by the World Health Organization (WHO) in March 2020, can be controlled with an effective COVID-19 vaccine (7). The COVID-19 presents with a continuum of respiratory tract symptoms such as fever, shortness of breath, pneumonia, influenzalike illness, etc., and is caused by the Severe Acute Respiratory Syndrome Coronavirus 2 (SARS-COV-2) $(8,9)$. In the U.S., the COVID-19 has resulted in 28,405,925 incident cases and 511,839 mortalities. Whereas, in Tennessee, $2.7 \%$ of total U.S. cases $(775,693)$ and $2.2 \%$ of total U.S. deaths $(11,421)$ have been recorded (10). Following mutations in their virus genome, new variants of the SARS-COV-2 have begun to emerge with alterations to their features. Concerningly, these genetic variants may increase disease severity and infectivity as well as change treatment and vaccine efficacy (11). Consequently, this ongoing public health crisis from COVID-19 has had devastating impacts on every aspect of human life causing significant morbidity and mortality, adverse psychological outcomes, and growing socioeconomic losses. Additionally, the pandemic has disrupted the hard-earned progress made in the last decade to improve vaccination rates. This current review sought to explore the barriers to pediatric vaccine uptake behaviors (e.g., vaccine hesitancy), as well as, propose recommendations for increasing vaccine confidence and compliance to immunization schedules within the context of the COVID-19 pandemic.

\section{VACCINATION COVERAGE AMONG CHILDREN (19-35 MONTHS) AND ADOLESCENTS (13-17 YEARS) IN TENNESSEE AND THE U.S.}

The CDC's Advisory Committee on Immunization Practice (ACIP) recommends routine immunizations against diseases e.g., measles, whooping cough for children ages through 2 years. For the 78.6 million children born between 1994 and 2013 in the U.S., routine childhood vaccinations have prevented an estimated 322 million illnesses, 21 million hospitalizations and 732,000 untimely deaths from measles $(70,748)$, varicella $(68,445)$, pertussis $(54,406)$, mumps $(42,704)$, and rubella $(36,540)(10)$. Consequently, within the last decade i.e. 20092017 , coverage for the combined 7 -vaccine series ${ }^{1}$ among children ages 19-35 months has risen comparably in the U.S. and Tennessee from $44.3 \%$ to $72.2 \%$ and $44.8 \%$ to $79.3 \%$, respectively (12) (Figure 1).

Concomitantly, among adolescents, vaccinations are recommended to prevent illnesses such as human papillomavirus (HPV), whooping cough, and meningococcal disease. As portrayed in Figure 2, HPV coverage; diphtheria, tetanus, and acellular pertussis (Tdap); and meningococcal conjugate vaccines have steadily increased in the last decade within the U.S. and Tennessee (12). Although, while the prevalence for all specified vaccines varied slightly within a $10 \%$-point range in 2008 , HPV vaccination rates have continued to significantly lag behind that of Tdap and meningococcal conjugate vaccines in recent years. While national vaccination rates for Tdap and meningococcal conjugate vaccines have reached or exceeded the HealthyPeople 2030 set-goal of $80 \%$ for vaccine coverage among adolescents (13 to 17 years), immunization with the HPV vaccine remains considerably low. See Figure 2. Despite current ACIP protocols and improving trends for other vaccines, the 2019 coverage for $\geq 1 \mathrm{HPV}$ vaccine among male and female adolescents was estimated at $71.5 \%$ in the US and 9.6 percentage points lower $(61.9 \%)$ in Tennessee (13).

\section{BARRIERS TO CHILDHOOD/ ADOLESCENT VACCINATION UPTAKE DURING THE COVID-19 PANDEMIC}

\section{Impacts of the COVID-19 Pandemic on Vaccination Rates}

As part of the efforts to "flatten the curve" and control the rapid spread of SARS-COV-2 during the COVID-19 pandemic, numerous policies and preventive public health measures including shelter-in-place, stay-at-home orders, social distancing, lockdowns, and other quarantine measures, were imposed (13). These precautionary measures, which have disrupted healthcare systems and health personnel services, have ultimately led to sub-optimal vaccine delivery services and vaccination rates (14). In the U.S., after the national emergency declaration, the aggregate count for pediatric vaccine doses procured by the Vaccine-for-Children (VFC) providers substantially declined (15). Similarly, the WHO recorded a 28-year reduction in global coverage for the Tdap

${ }^{1} \geq 4$ doses of diphtheria, tetanus toxoid and pertussis, $\geq 3$ doses of polio, $\geq 1$ measles-containing vaccine, Haemophilus influenza type $b$ full series, $\geq 3$ hepatitis $b, \geq 1$ varicella and $\geq 4$ pneumococcal vaccine. 


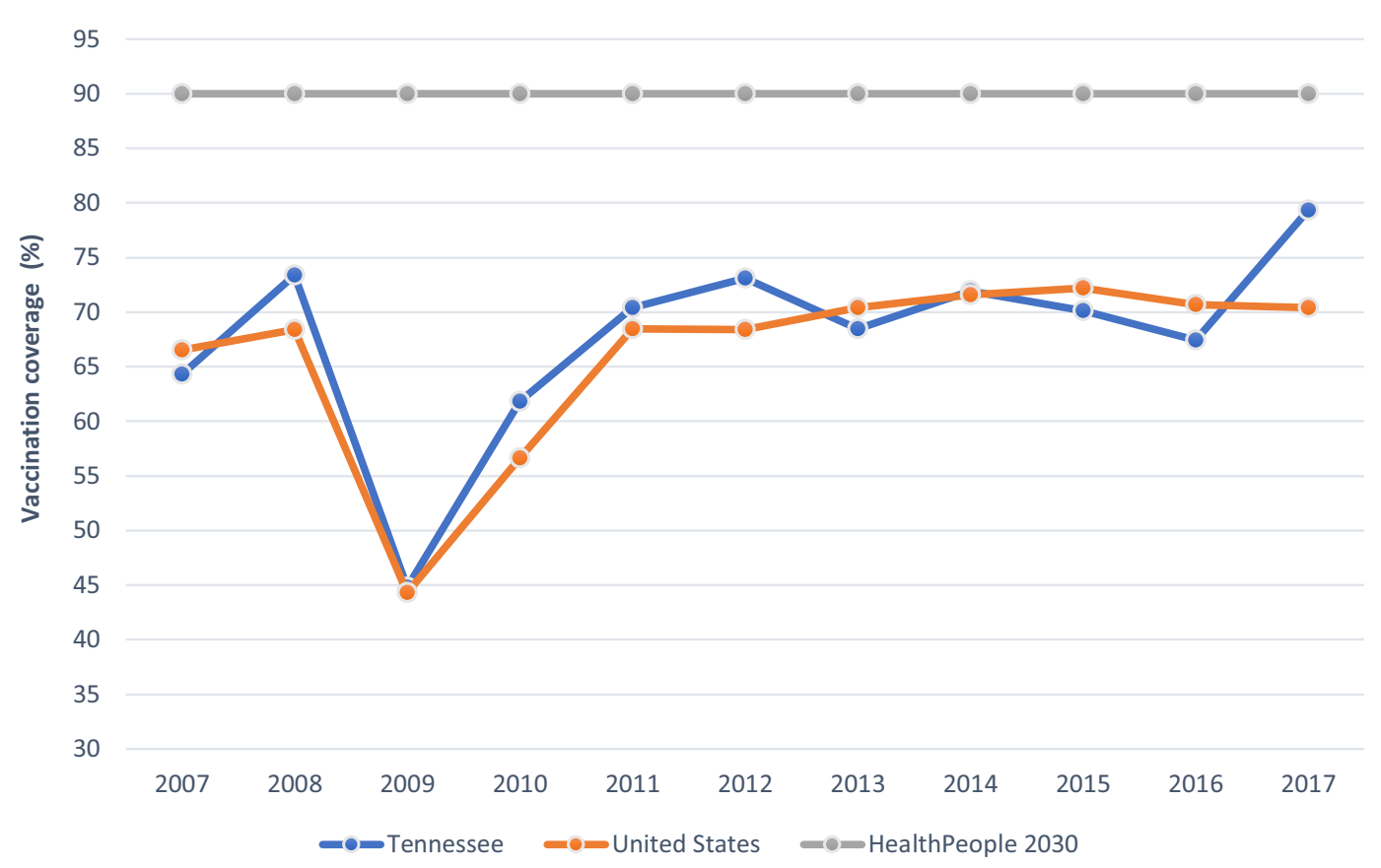

FIGURE 1 | Combined 7-Vaccine Series Coverage (\%) by Year among Children ages 19-35 months in Tennessee and the United States in Relation to the HealthyPeople2030 Goal.Combined 7-vaccine series: $\geq 4$ doses of diphtheria, tetanus toxoid, and pertussis, $\geq 3$ doses of polio, $\geq 1$ measles-containing vaccine, influenza $1 \mathrm{~b}$ full series, $\geq 3$ hepatitis b, $\geq 1$ varicella and $\geq 4$ pneumococcal vaccine. Data source: National Center for Immunization and Respiratory Diseases. Retrieved October 13, 2020

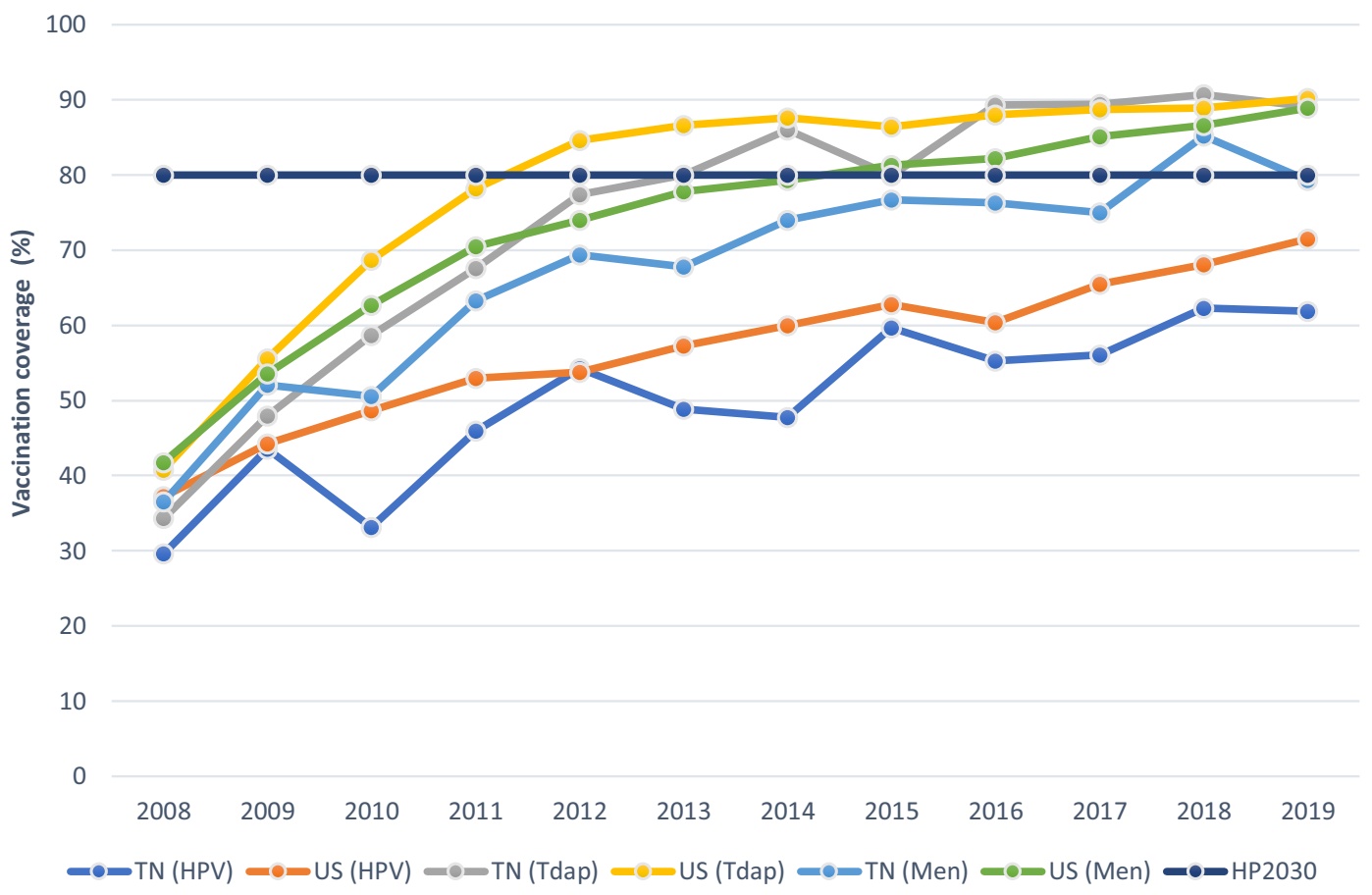

FIGURE 2 | Estimated Vaccine Coverage (\%) by Year among Adolescents ages 13-17 years in Tennessee and the United States in Relation to the HealthyPeople2030 Goal.HPV= human papillomavirus vaccine; Tdap= tetanus toxoid, reduced diphtheria toxoid, and acellular pertussis vaccine; Men= meningococcal conjugate vaccine, TN=Tennessee, US=United States. HPV, Tdap, and meningococcal conjugate vaccinations are depicted as the receipt of $\geq 1$ dose of vaccine Data source: National Center for Immunization and Respiratory Diseases. Retrieved October 13, 2020 
vaccine (16). Also, notable was the collective shift in focus on routine vaccinations to respond urgently to the ongoing health crisis (17). These events have been exacerbated by the adverse rippling effects of other COVID-19 pandemic sequelae which include overwhelmed healthcare systems; inequalities in healthcare delivery; financial recession and job losses; worries about vaccine costs; inadequate personal protective equipment for healthcare workers; severe shortages in testing modalities and treatment therapies; long-term school closures; contradictory messages from health agencies/authorities; as well as; disruptions to transportation and travel restrictions. Additionally, parental concerns regarding exposure to the COVID-19 have discouraged individuals who would otherwise have utilized vaccination services, thus, resulting in postponed/ canceled medical appointment visits. Moreover, restrictions on routine in-person office visits due to physical distancing protocols have limited health care providers' communications promoting vaccine uptake to patients (18).

Overall, the existing COVID-19 pandemic has forestalled the painstaking but incremental progress made in the last decade to improve uptake for HPV and other vaccines. Disruptions to vaccine delivery services have negatively impacted timely immunizations leaving children/adolescents susceptible to vaccine-preventable diseases. As the United Nations Children's Fund (UNICEF) Executive Director Henrietta Fore aptly describes it, "COVID-19 has made previously routine vaccination a daunting challenge..." (16). An outbreak of vaccine-preventable diseases during the COVID-19 pandemic would only worsen the already strained health care system due to rising hospitalization and death rates.

\section{Impacts of Social Determinants of Health on Vaccination Rates}

In research, individual and interpersonal level approaches have long been utilized to examine and facilitate behavior change. However, this approach is limited as it fails to integrate societal components that influence health outcomes. More recently, factors that facilitate and/or hinder the implementation of health behaviors are addressed using a novel approach - Social Determinants of Health (SDoH) (19). The SDoH encompasses elements in an individual's neighborhood, community, and environment as determined by where that individual is born, resides, learns, works, worships, etc. Consequently, the HealthyPeople 2030 SDoH Framework classifies SDoH indicators into five categories: social and community context, education, economic stability, neighborhood, and built environment, and health and health care (19). SDoH include access to education, affordable housing and health services, public safety, food security, etc. (20).

$\mathrm{SDoH}$ are impacted by the distribution of resources that improve the quality of life and public health outcomes. For instance, in the U.S., individuals who reside in certain metropolitan statistical areas (MSA), non-MSA (mostly rural), and without health insurance are disproportionately less likely to be vaccinated (21). Additionally, parental education; household living conditions and income; healthcare access; philosophical and cultural beliefs; religious affiliations; and urban Vs. rural residence, are some of the $\mathrm{SDoH}$ that influence childhood vaccination rates (22). In recent times, the COVID-19 pandemic has underscored the importance of incorporating $\mathrm{SDoH}$ into health systems and health service delivery. While only a few studies have examined the impacts of $\mathrm{SDoH}$ on vaccinations, it is likely the COVID-19 pandemic has exacerbated the adverse effects of some $\mathrm{SDoH}$ on vaccination uptake behaviors e.g., employment, poverty, healthcare access, food insecurity, education, etc.

\section{Impacts of the Vaccine Hesitancy/Refusal on Vaccination Rates}

The WHO describes vaccine hesitancy as the, "delay in acceptance or refusal of vaccines despite availability of vaccine services" and categorizes it within the top ten threats to global health (23). This phenomenon also incorporates the antivaccine movement as well as parents' adoption of alternate, nonstandardized vaccination schedules. Parent's hesitancy, refusals, and delays in adhering to routine childhood immunizations are largely responsible for a significant number of unvaccinated/ under-vaccinated children, disease outbreaks, co-morbidities (e.g., meningitis, pneumonia, HPV-related cancers), as well as, untimely deaths. Vaccine hesitancy and refusal have mostly occurred due to state/local policies that have allowed parents to decline routine childhood vaccinations based on non-medical exemptions (24). These non-medical exemptions occur in the form of religious exemptions e.g., due to an individual's religious beliefs which oppose the use of fetal tissue for vaccines and personal belief exemptions e.g., due to an individual's logical reasoning which disapproves the use of non-natural products for vaccines (24). Prevalence estimates for vaccination exemptions are currently $2.5 \%$ and $1.9 \%$ nationally and in Tennessee, respectively (25). Currently, in the U.S., 45 states and Washington D.C. permit religious exemptions while 15 states allow philosophical exemptions from childhood vaccinations.

Due to parental concerns on vaccine safety/side effects, some studies which implied a link between the measles-mumps-rubella (MMR) vaccine and autism, played a significant role in vaccine hesitancy and refusal $(26,27)$. However, Wakefield et al. was retracted due to methodological deficits and data misrepresentation (28). Following larger multiple studies and a wealth of scientific evidence, this hypothesized link was disproved and the safety of the MMR vaccine reinforced (29, 30). Likewise, fears regarding administering multiple vaccines concurrently in a child, unverified sources, and misinformation campaigns from the internet/media have served to dissuade parents from seeking child vaccination services (22). Nevertheless, evidence-focused literature has debunked numerous myths and misinformation citing that recommended vaccines are too many; contain unfavorable ingredients e.g., mercury, aluminum, DNA fragments; damage immune and neurologic systems; and display life-threatening side-effects, etc. (31). 


\section{IMPLICATIONS OF NON-ADHERENCE TO VACCINATION PROTOCOLS}

Overall, a major accomplishment of universal vaccine coverage has been to markedly reduce and/or eradicate transmittable diseases that would ultimately have led to premature mortalities in the pre-vaccine era. Despite these advances, however, sporadic outbreaks within communities have continued to occur and coincide with pockets of low community vaccination rates and limited ability for vaccines to elicit immune responses (32). The majority of recent outbreaks have occurred among unvaccinated individuals particularly those exposed to illnesses imported from other countries, as well as those who claimed religious or personal exemptions or had missed immunization opportunities $(33,34)$.

As a result, outbreaks, incidence, prevalence, and transmission of illnesses e.g., measles virus are seeing an increasing trend in the U.S. (35). Between January and December 2019, there were 1,282 confirmed cases of measles reported in 31 states. This is significantly higher than the 375 cases seen in 2018 and represents the highest prevalence reported since 1992 (36). Moreover, in 2019, almost half of the 14 counties that granted non-medical vaccination exemptions to parents of kindergarten school-aged children (37) experienced the measles outbreak (38). Accounts of other vaccine-preventable outbreaks have occurred for the Haemophilus influenza type B (39) and pneumococcal infections (40).

In addition, adolescents engaging in risky sexual behaviors e.g., multiple sexual partners, and unprotected sexual intercourse, are susceptible and considered high risk for acquiring HPV infections (41). Between 2013 and 2017, there were an estimated 45,300 HPVassociated cancers recorded consisting of cervical $(12,143)$, oropharyngeal $(19,775)$, and anal $(7,083)$ cancers $(42)$. More than $90 \%$ of all HPV-associated cancers (e.g., cervical, vulvar, vaginal, and anal cancers) are preventable through receipt of the HPV vaccine (43).

\section{RECOMMENDATIONS TO RESTORE PARENTAL VACCINE CONFIDENCE DURING THE COVID-19 PANDEMIC}

Despite the disruption to health amenities during the COVID-19 pandemic, the continuity of immunization services for children and adolescents is pertinent to enable progress in vaccination trends as well as deter vaccine-preventable diseases and outbreaks. Against the backdrop of the COVID-19 pandemic and vaccine non-compliance/refusal, the pediatrician and other healthcare providers are uniquely qualified to promote vaccinations achieved through the use of strong, presumptive languaging (44) to offer effective, consistent recommendations that emphasize disease/cancer prevention. The healthcare professionals' reluctance to share recommendations that facilitate vaccine uptake could result in parental hesitancy, refusal, and delay. In 2019, national HPV coverage among adolescents with a provider's recommendation (74.7\% CI:73.376.0) was almost twice as those without one (46.7\% CI:43.8-49.6)
(21). Concurrently, in Tennessee, coverage was 72.4\% (CI:64.379.2) for those who received advice from their providers as opposed to those without (28.2\% CI:16.6-43.6) (21). This supports the notion that recommendations offered by health providers could significantly predict vaccine uptake, thereby reinforcing the need for personalized patient-provider interactions.

Consequently, interventions and training should empower healthcare providers to disseminate evidence-based advice on vaccines. Specifically, quality improvement coaching such as the CDC's Assessment, Feedback, Incentives, and eXchange (AFIX) program which facilitates provider's education and feedback through face-to-face coaching has been shown to improve immunization rates (45). Also, campaigns should aim to increase providers' self-efficacy and confidence to address parental concerns on vaccine's efficacy, side effect(s), lack of health insurance as well as adopt the use of electronic medical records (EMRs), immunization information systems, and medical practice alerts to remind parents about scheduled regular in-patient visits (46). Parents without health insurance should receive information on reduced out-of-pocket costs and publicly-funded vaccines available through the VFC program (13). For parents with religious or philosophical beliefs, healthcare provider's information on the fewer components of proteins and polysaccharides in vaccines could serve to allay fears (38). For others, communication on societal norms that promote routine vaccination as a social responsibility could increase vaccine uptake (47). Moreover, addressing parental concerns for needle pain, skin reactions, and sensitivity as well as the adoption of motivational interview techniques (i.e. acceptance, compassion, collaboration, etc.) could be impactful (38).

Furthermore, best-practices that facilitate adherence to standardized safety protocols, beneficence, and non-transmission of the COVID-19 should be employed e.g., physical distancing, mask usage, hand hygiene practices, etc. Training and instructions on disease/infection prevention and control should be incorporated into the continuing medical education (CME) curriculum for health professionals (48). Wellness-child visits through telemedicine video conferencing; administering of vaccines through outdoor/curbside/ drive-through services; vaccine delivery in alternative settings e.g., pharmacies, schools; minimizing on-site patient visit at any single point in time; delineating specific, well-ventilated rooms for wellness visits, vaccine-only visits, etc., should be implemented to tackle the current COVID-19 health crisis (14). Overall, clinicians should work in synergy with other healthcare team members to maximize scheduled wellness/immunization visits, and other routine medical checkups particularly in places with a low prevalence of health provider's recommendations e.g., rural areas. While mandatory vaccination policies have been shown to be associated with higher vaccine acceptance rates (49), these should be reinforced with patient-provider interactions that address parental concerns. Additionally, nonmedical exemption laws should be reviewed to ensure that in places where they have not been prohibited, there should be in place effective administrative controls so that exemptions do not become easier defaults when compared to vaccinations $(50,51)$. Government health officials, as 
well as, the school districts should continue to enforce and maintain up-to-date immunization records. The catch-up vaccination protocols issued by the CDC to facilitate coverage for children with missed appointments during the pandemic should be implemented (52). Education campaigns should also be tailored to engage local and religious leaders, be culturally appropriate and address specific concerns from vaccine-hesitant populations.

Ultimately, a multifaceted, multidisciplinary approach involving science, engineering, and social sciences should be incorporated to explore facilitators and barriers to childhood vaccine uptake as well as comprehend the drivers for vaccine hesitancy, refusal, and delay. Accordingly, the application of Machine Learning and Artificial Intelligence (53) would be beneficial to (a) identify trends, patterns, and prevalence of childhood vaccine uptake and vaccine-preventable illnesses; (b) investigate psychosocial factors and disparities influencing the receipt of vaccines; as well as (c) examine the interface between vaccine-preventable disease outbreaks and vaccine hesitancy/ refusal. Specifically, more concerted efforts should be made to implement Personal Health Libraries (54) along with Public Health Observatories (55) for vaccine acceptance surveillance (56) on a national scale and within the state of Tennessee. These intelligent tools can facilitate precision health promotion to increase vaccination rates (57) as well as examine causal associations between predictors (e.g., SDoH, COVID-19 pandemic policies, etc.) and outcomes (e.g., vaccine uptake,

\section{REFERENCES}

1. Kazi AM. The role of mobile phone-based interventions to improve routine childhood immunisation coverage. Lancet Global Health (2017) 5(4):e377-8. doi: 10.1016/S2214-109X(17)30088-8

2. Centers for Disease Control and Prevention. Measles: History of measles (2018). Available at: https://www.cdc.gov/measles/about/history.html (Accessed March 1st, 2021).

3. Centers for Disease Control and Prevention. Vaccines and Immunizations: What Would Happen If We Stopped Vaccinations (2018). Available at: https:// www.cdc.gov/vaccines/vac-gen/whatifstop.htm (Accessed March 1st, 2021).

4. Anderson EL. Recommended solutions to the barriers to immunization in children and adults. Missouri Med (2014) 111(4):344.

5. Whitney CG, Zhou F, Singleton J, Schuchat A. Benefits from immunization during the vaccines for children program era-United States, 1994-2013. MMWR Morbid Mortal Wkly Rep (2014) 63(16):352.

6. Dreyzehner J, Reagan D, Jones TF. Tennessee Department of Health: Communicable and Environmental Diseases and Emergency Preparedness (2012). Available at: https://www.tn.gov/content/dam/tn/health/documents/ cedep-weeklyreports/AnnualReport2010-12.pdf (Accessed March 1st, 2021).

7. van Riel D, de Wit E. Next-generation vaccine platforms for COVID-19. Nat Mater (2020) 19(8):810-2. doi: 10.1038/s41563-020-0746-0

8. Yuki K, Fujiogi M, Koutsogiannaki S. COVID-19 pathophysiology: A review. Clin Immunol (2020) 20:108427. doi: 10.1016/j.clim.2020.108427

9. Bonam SR, Kotla NG, Bohara RA, Rochev Y, Webster TJ, Bayry J. Potential immuno-nanomedicine strategies to fight COVID-19 like pulmonary infections. Nano Today (2020) 4:101051. doi: 10.1016/j.nantod.2020.101051

10. Centers for Disease Control and Prevention. Coronavirus Disease 2019 (COVID19). CDC COVID Data Tracker (2020). Available at: https://covid.cdc.gov/coviddata-tracker/\#cases_casesper100klast7days (Accessed March 1st, 2021).

11. Center for Disease Control and Prevention. COVID-19: US COVID-19 cases caused by variants (2021). Available at: https://www.cdc.gov/coronavirus/ 2019-ncov/transmission/variant-cases.html (Accessed March 1st, 2021). vaccine hesitancy). In addition to facilitating linkages between healthcare systems, these applications could ensure timely access to accurate health information crucial for effective decisionmaking regarding vaccine access, allocation services, etc. Health policy-driven changes that address vaccine hesitancy, $\mathrm{SDoH}$ inequalities, and disparities in vaccination access would be advantageous. Finally, more research that qualitatively examines barriers to vaccine uptake behaviors, as well as drivers to vaccine hesitancy among specific populations, would be beneficial.

\section{AUTHOR CONTRIBUTIONS}

OO: writing original draft, review and editing, visualization, and conceptualization. $\mathrm{RB}$ and $\mathrm{RD}$ : review and editing, and conceptualization. AS-N: review and editing, conceptualization, obtained funding, and supervision. All authors contributed to the article and approved the submitted version.

\section{FUNDING}

This study is partially supported by Grant\# 1R37CA23411901A1 from National Cancer Institute (NCI).
12. Center for Disease Control and Prevention. through 2017 childhood combined 7-vaccine series coverage trend report (2018). Available at: https://www.cdc. gov/vaccines/imz-managers/coverage/childvaxview/data-reports/7-series/ trend/index.html (Accessed March 1st, 2021).

13. Elam-Evans LD, Yankey D, Singleton JA, Sterrett N, Markowitz LE, Williams CL, et al. National, regional, state, and selected local area vaccination coverage among adolescents aged 13-17 years-United States, 2019. Morbid Mortal Wkly Rep (2020) 69(33):1109. doi: 10.15585/mmwr.mm6933al

14. Bramer CA, Kimmins LM, Swanson R, Kuo J, Vranesich P, Jacques-Carroll LA, et al. Decline in child vaccination coverage during the COVID-19 pandemic-Michigan Care Improvement Registry, May 2016-May 2020. Am J Transplant (2020) 20(7):1930. doi: 10.1111/ajt.16112

15. Santoli JM. Effects of the COVID-19 pandemic on routine pediatric vaccine ordering and administration-United States, 2020. MMWR. Morbid Mortal Wkly Rep (2020) 69:591-3. doi: 10.15585/mmwr.mm6919e2

16. World Health Organization. WHO and UNICEF warn of a decline in vaccinations during COVID-19 (2020). Available at: https://www.who.int/ news/item/15-07-2020-who-and-unicef-warn-of-a-decline-in-vaccinationsduring-covid-19 (Accessed March 1st, 2021).

17. Saxena S, Skirrow H, Bedford H. Routine vaccination during covid-19 pandemic response. BMJ (2020) 369. doi: 10.1136/bmj.m2392

18. Gilkey MB, Bednarczyk RA, Gerend MA, Kornides ML, Perkins RB, Saslow D, et al. Getting human papillomavirus vaccination back on track: Protecting our national investment in human papillomavirus vaccination in the COVID-19 era. J Adolesc Health (2020) 67(5):633-4. doi: 10.1016/j.jadohealth.2020.08.013

19. Thompson EL, Rosen BL, Maness SB. Social determinants of health and human papillomavirus vaccination among young adults, National Health Interview Survey 2016. J Community Health (2019) 44(1):149-58. doi: 10.1007/s10900-018-0565-2

20. US Department of Health and Human Services. Social determinants of health (2014). Available at: https://www.healthypeople.gov/2020/topics-objectives/ topic/social-determinants-of-health (Accessed March 1st, 2021).

21. Walker TY, Elam-Evans LD, Yankey D, Markowitz LE, Williams CL, Fredua $\mathrm{B}$, et al. National, regional, state, and selected local area vaccination coverage 
among adolescents aged 13-17 years-United States. Morbid Mortal Wkly Rep (2019) 68(33):718. doi: 10.15585/mmwr.mm6833a2

22. Glatman-Freedman A, Nichols K. The effect of social determinants on immunization programs. Hum Vaccines Immunother (2012) 8(3):293-301. doi: 10.4161/hv.19003

23. World Health Organization. Report of the SAGE working group on vaccine hesitancy (2014). Available at: https://www.who.int/immunization/sage/ meetings/2014/october/1_Report_WORKING_GROUP_vaccine_hesitancy_ final.pdf (Accessed March 1st, 2021).

24. Phadke VK, Bednarczyk RA, Salmon DA, Omer SB. Association between vaccine refusal and vaccine-preventable diseases in the United States: a review of measles and pertussis. Jama (2016) 315(11):1149-58. doi: 10.1001/ jama.2016.1353

25. Seither R. Vaccination Coverage with Selected Vaccines and Exemption Rates Among Children in Kindergarten-United States, 2019-20 School Year. MMWR Morbid Mortal Wkly Rep (2021) 70:75-82. doi: 10.15585/ mmwr.mm7003a2

26. Singh VK, Lin SX, Yang VC. Serological association of measles virus and human herpesvirus-6 with brain autoantibodies in autism. Clin Immunol Immunopathol (1998) 89(1):105-8. doi: 10.1006/clin.1998.4588

27. Wakefield AJ, Murch SH, Anthony A, Linnell J, Casson DM, Malik M, et al. RETRACTED: Ileal-lymphoid-nodular hyperplasia, non-specific colitis, and pervasive developmental disorder in children. Lancet (1998) 351:637-41. doi: 10.1016/S0140-6736(97)11096-0

28. Leask J, Booy R, McIntyre PB. MMR, Wakefield and The Lancet: what can we learn. Med J Aust (2010) 193(1):5-7. doi: 10.5694/j.1326-5377.2010.tb03730.x

29. Madsen KM, Hviid A, Vestergaard M, Schendel D, Wohlfahrt J, Thorsen P, et al. A population-based study of measles, mumps, and rubella vaccination and autism. New Engl J Med (2002) 347(19):1477-82. doi: 10.1056/ NEJMoa021134

30. Taylor B, Miller E, Farrington C, Petropoulos MC, Favot-Mayaud I, Li J, et al. Autism and measles, mumps, and rubella vaccine: no epidemiological evidence for a causal association. Lancet (1999) 353(9169):2026-9. doi: 10.1016/S0140-6736(99)01239-8

31. Geoghegan S, O'Callaghan KP, Offit PA. Vaccine safety: myths and misinformation. Front Microbiol (2020) 11:372. doi: 10.3389/fmicb.2020.00372

32. Hebert CJ, Hall CM, Odoms LN. Lessons learned and applied: what the 20th century vaccine experience can teach us about vaccines in the 21st century. Hum Vaccines Immunother (2012) 8(5):560-8. doi: 10.4161/hv.19204

33. Hall V, Banerjee E, Kenyon C, Strain A, Griffith J, Como-Sabetti K, et al. Measles outbreak-Minnesota April-May 2017. MMWR Morbid Mortal Wkly Rep (2017) 66(27):713. doi: 10.15585/mmwr.mm6627al

34. McDonald R, Ruppert PS, Souto M, Johns DE, McKay K, Bessette N, et al. Notes from the field: measles outbreaks from imported cases in Orthodox Jewish communities-New York and New Jersey, 2018-2019. Morbid Mortal Wkly Rep (2019) 68(19):444. doi: 10.15585/mmwr.mm6819a4

35. Phadke VK, Bednarczyk RA, Omer SB. Vaccine Refusal and Measles Outbreaks in the US. Jama (2020) 324(13):1344-5. doi: 10.1001/jama.2020.14828

36. Centers for Disease Control and Prevention. Measles: Measles cases and outbreaks (2020). Available at: https://www.cdc.gov/measles/cases-outbreaks. html (Accessed March 1st, 2021).

37. Olive JK, Hotez PJ, Damania A, Nolan MS. The state of the antivaccine movement in the United States: A focused examination of nonmedical exemptions in states and counties. PloS Med (2018) 15(6):e1002578. doi: 10.1371/journal.pmed.1002578

38. Hotez PJ, Nuzhath T, Colwell B. Combating vaccine hesitancy and other 21st century social determinants in the global fight against measles. Curr Opin Virol (2020) 41:1-7. doi: 10.1016/j.coviro.2020.01.001

39. Rainbow J, Danila R, Bahta L, White K, Ehresmann K, Lynfield R, et al. Invasive Haemophilus influenzae type B disease in five young childrenMinnesota, 2008. Morbid Mortal Wkly Rep (2009) 58(3):58-60.

40. Glanz JM, McClure DL, O’Leary ST, Narwaney KJ, Magid DJ, Daley MF, et al. Parental decline of pneumococcal vaccination and risk of pneumococcal related disease in children. Vaccine (2011) 29(5):994-9. doi: 10.1016/ j.vaccine.2010.11.085

41. Shannon CL, Klausner JD. The growing epidemic of sexually transmitted infections in adolescents: a neglected population. Curr Opin Pediatr (2018) 30 (1):137. doi: 10.1097/MOP.0000000000000578
42. Centers for Disease Control and Prevention. Cancers associated with human papillomavirus, United States-2013-2017 (2020). Available at: https://www. cdc.gov/cancer/uscs/about/data-briefs/no18-hpv-assoc-cancers-UnitedStates2013-2017.htm (Accessed March 1st, 2021).

43. Printz C. FDA approves Gardasil 9 for more types of HPV. Cancer (2015) 121 (8):1156-7. doi: 10.1002/cncr.29374

44. Jacobson RM, St. Sauver JL, Griffin JM, MacLaughlin KL, Finney Rutten LJ. How health care providers should address vaccine hesitancy in the clinical setting: Evidence for presumptive language in making a strong recommendation. Hum Vaccines Immunother (2020) 16(9):2131-5. doi: $10.1080 / 21645515.2020 .1735226$

45. Calo WA, Gilkey MB, Leeman J, Heisler-MacKinnon J, Averette C, Sanchez S, et al. Coaching primary care clinics for HPV vaccination quality improvement: Comparing in-person and webinar implementation. Trans Behav Med (2019) 9(1):23-31. doi: 10.1093/tbm/iby008

46. Ventola CL. Immunization in the United States: recommendations, barriers, and measures to improve compliance: part 1: childhood vaccinations. Pharm Ther (2016) 41(7):426.

47. Brewer NT, Chapman GB, Rothman AJ, Leask J, Kempe A. Increasing vaccination: putting psychological science into action. psychol Sci Public Interest (2017) 18(3):149-207. doi: 10.1177/1529100618760521

48. World Health Organization \& United Nations Children's Fund (UNICEF. Immunization in the context of COVID-19 pandemic: frequently asked questions (FAQ) (2020). Available at: https://apps.who.int/iris/handle/10665/ 331818 (Accessed March 1st, 2021).

49. Vaz OM, Ellingson MK, Weiss P, Jenness SM, Bardají A, Bednarczyk RA, et al. Mandatory vaccination in Europe. Pediatrics (2020) 145(2). doi: 10.1542/ peds.2019-0620

50. Omer SB, Porter RM, Allen K, Salmon DA, Bednarczyk RA. Trends in kindergarten rates of vaccine exemption and state-level policy, 2011-2016. InOpen Forum Infect Dis (2018) 5(2):ofx244. doi: 10.1093/ofid/ofx244

51. Omer SB, Pan WK, Halsey NA, Stokley S, Moulton LH, Navar AM, et al. Nonmedical exemptions to school immunization requirements: secular trends and association of state policies with pertussis incidence. Jama (2006) 296 (14):1757-63. doi: 10.1001/jama.296.14.1757

52. Centers for Disease Control and Prevention. Immunization Schedules: Vaccination Catch-Up Guidance (2020). Available at: https://www.cdc.gov/ vaccines/schedules/hcp/schedule-changes.html (Accessed March 1st, 2021).

53. Shaban-Nejad A, Michalowski M, Buckeridge DL. Health intelligence: how artificial intelligence transforms population and personalized health. NPJ Digit Med (2018) 1:53. doi: 10.1038/s41746-018-0058-9

54. Ammar N, Bailey J, Davis RL, Shaban-Nejad A. The Personal Health Library: A Single Point of Secure Access to Patient Digital Health. Stud Health Technol Inf (2020) 270:448-52. doi: 10.3233/SHTI200200

55. Brakefield WS, Ammar N, Olusanya O, Ozdenerol E, Thomas F, Stewart AJ, et al. Implementing an Urban Public Health Observatory for (Near) RealTime Surveillance for the COVID-19 Pandemic. Stud Health Technol Inform (2020) 275:22-6. doi: 10.3233/SHTI200687

56. Brien S, Naderi N, Shaban-Nejad A, Mondor L, Kroemker D, Buckeridge DL. Vaccine attitude surveillance using semantic analysis: constructing a semantically annotated corpus. In: . WWW "13 Companion: Proceedings of the 22nd International Conference on World Wide Web (2013) ACM Press. p. 683-6. doi: 10.1145/2487788.2488023

57. Shaban-Nejad A, Michalowski M, Peek N, Brownstein JS, Buckeridge DL. Seven pillars of precision digital health and medicine. Artif Intell Med (2020) 103:101793. doi: 10.1016/j.artmed.2020.101793

Conflict of Interest: The authors declare that the research was conducted in the absence of any commercial or financial relationships that could be construed as a potential conflict of interest.

Copyright (c) 2021 Olusanya, Bednarczyk, Davis and Shaban-Nejad. This is an openaccess article distributed under the terms of the Creative Commons Attribution License (CC BY). The use, distribution or reproduction in other forums is permitted, provided the original author(s) and the copyright owner(s) are credited and that the original publication in this journal is cited, in accordance with accepted academic practice. No use, distribution or reproduction is permitted which does not comply with these terms. 